ist recht gut. In der metallischen Phase wurde der Ladungsträgeranteil nach $W_{\text {IEDEMANN-F }}$ RANz berechnet und von der gemessenen totalen Wärmeleitfähigkeit abgezogen, um den Gitteranteil zu erhalten. Die Gitterwärmeleitung nimmt danach am Umwandlungspunkt Halbleiter-Metall um etwa einen Faktor 4 zu, was wohl darauf zurückzuführen ist, daß es sich um eine Umwandlung von einer niedersymmetrischen $\beta$-Phase, deren Struktur nicht eindeutig bestimmt werden konnte, zu einer hochsymmetrischen kubischen $\alpha$-Phase handelt.

$\mathrm{Da}$ man den oben erwähnten Unterschied in den
Breiten der verbotenen Zone und in den Beweglichkeitsverhältnissen in der Wärmeleitung nicht bemerkt, ist darauf zurückzuführen, daß der Ladungsträgeranteil für die verschiedenen Phasen gerade gleich groß ist.

Die Effektivität der Probe mit $1,5 \cdot 10^{18}$ Störstellen pro $\mathrm{cm}^{3}$ zeigt ein flaches Maximum bei $0{ }^{\circ} \mathrm{C}$ von $1,4 \cdot 10^{-3}{ }^{\circ} \mathrm{K}^{-1}$.

Für die Gewährung finanzieller Mittel aus dem Arbeitsbeschaffungskredit des Bundes sind wir der Eidg. Kommission zur Förderung der wissenschaftlichen Forschung sehr zu Dank verpflichtet.

\title{
Zur Theorie des p-n-Übergangs bei Berüdksichtigung der Rekombination im Übergangsgebiet
}

\author{
Von H. Diehl \\ Aus dem Institut für Struktur der Materie der Universität Marburg a. d. Lahn \\ (Z. Naturforschg. 17 a, 889-899 [1962] ; eingegangen am 8. August 1962)
}

\begin{abstract}
Bisher zur Theorie des $\mathrm{p}-\mathrm{n}$-Übergangs erschienene Arbeiten gehen von der Annahme aus, daß die elektrochemischen Potentiale im Übergangsgebiet nahezu konstant sind. Damit ist der Gültigkeitsbereich dieser Theorien auf große Diffusionslängen beschränkt, ohne daß jedoch eine genauere Grenze angegeben werden konnte. Eine solche Angabe wird durch die vorliegende Arbeit ermöglicht. Ausgehend von den Stromgleichungen für Elektronen und Löcher wird ein allgemeiner Formalismus entwickelt, der zu einem geschlossenen Ausdruck für die elektrochemischen Potentiale führt. Diese Überlegungen werden auf den abrupten symmetrischen $\mathrm{p}-\mathrm{n}$-Übergang spezialisiert und für ein konkretes Beispiel numerisch ausgewertet. Es zeigt sich, daß die Anwendbarkeit der obengenannten Theorien auf Diffusionslängen begrenzt ist, die größer sind als die Breite des Übergangsgebietes im unbelasteten Fall. Außerdem wird die Änderung der Strom-Spannungs-Charakteristiken mit abnehmender Diffusionslänge diskutiert und so die Verbindung zu dem in der Literatur bekannten Grenzfall verschwindender Diffusionslänge hergestellt. Die durchgeführten Untersuchungen umfassen die früheren Rechnungen als Spezialfälle und zeigen im wesentlichen Übereinstimmung mit deren Ergebnissen.
\end{abstract}

Die ersten theoretischen Untersuchungen des p-n-Übergangs stammen von SHockley ${ }^{1}$. Sie beschränken sich auf den Fall geringer Rekombination, die im Übergangsgebiet vernachlässigt wird. Die durchgeführten Rechnungen liefern den Strom

$$
i=i_{\mathrm{s}}\left(e^{q V /(k T)}-1\right) ;
$$

$i_{\mathrm{S}}$ ist der Sättigungsstrom bei Sperrbelastung, $q$ die Elementarladung, $V$ die angelegte Spannung, $k$ die Boltzmann-Konstante, $T$ die absolute Temperatur.

Die Strom-Spannungs-Charakteristik eines $\mathrm{p}-\mathrm{n}$ Übergangs in Germanium bei Zimmertemperatur wird durch diese Theorie gut beschrieben. Gehen wir jedoch zu tiefen Temperaturen über oder be-

1 W. Shоскцеу, Bell. Syst. Tech. J. 28, 435 [1949].

2 M. Bernard, J. Electronics 2, 579 [1957]. trachten einen $\mathrm{p}-\mathrm{n}$-Übergang in Silicium, so zeigen die theoretischen und experimentellen Ergebnisse im allgemeinen keine Übereinstimmung. In diesen Fällen ist die Vernachlässigung der Rekombination im Übergangsgebiet nicht zulässig, denn der durch sie bedingte Strom wächst mit abnehmender Eigenleitungskonzentration $n_{\mathrm{i}}$, die bei Silicium um den Faktor $10^{3}$ kleiner ist als bei Germanium und um so geringer wird, je tiefer die Temperatur ist.

Eine Berücksichtigung der Rekombination im Übergangsgebiet erfolgt bei Bernard ${ }^{2}$, SAH, Noyce und Shockley ${ }^{3}$ sowie Chevychelov ${ }^{4}$. Die drei Arbeiten beschränken sich auf $p-n$-Übergänge, deren

\footnotetext{
3 C. T. SAH, R. N. Noyce u. W. Shockley, Proc. Inst. Radio Engrs. 45, 1228 [1957].
}

4 A. D. Chevychelov, Soviet Phys.-Solid State 1, 1102 [1960]. 
elektrochemische Potentiale im Übergangsgebiet als konstant angesehen werden können, was nach BERNARD bei hinreichend großen Diffusionslängen und entsprechend geringer Rekombination der Fall ist. Die berechneten Strom-Spannungs-Charakteristiken zeigen weitgehende Übereinstimmung, obwohl verschiedene Ansätze für das elektrostatische Potential verwendet sind. Wir können daraus schließen, daß die Potentialform relativ unempfindlich in die Rechnung eingeht.

Der $\mathrm{p}-\mathrm{n}$-Übergang bei verschwindender Lebensdauer der Ladungsträger wird von Madelung ${ }^{5}$ behandelt. Die Rekombination ist unendlich hoch, so daß die elektrochemischen Potentiale für Elektronen und Löcher zusammenfallen. Das Verfahren, nach dem die Strom - Spannungs-Charakteristik ermittelt wird, beruht auf einer Analogie zur Scнотткуschen Randschichttheorie. Es liegt der zur SHockLeyschen Diffusionstheorie ${ }^{1}$ entgegengesetzte Grenzfall vor.

In der vorliegenden Arbeit soll nun untersucht werden, welches Aussehen die Strom-SpannungsCharakteristiken von $\mathrm{p}-\mathrm{n}$-Übergängen haben, die durch die existierenden Theorien nicht beschrieben werden. Insbesondere interessiert die Frage, wie sich die Charakteristiken ändern, wenn die Rekombination zunimmt und so groß wird, daß die Annahme konstanter elektrochemischer Potentiale im Übergangsgebiet nicht mehr gilt. Wir beginnen unsere Untersuchung mit der Berechnung eines geschlossenen Ausdruckes für den Verlauf der elektrochemischen Potentiale in Abhängigkeit von der Ortskoordinate $x$ und haben so die Möglichkeit, in konkreten Fällen durch numerische Auswertung den Gültigkeitsbereich der bestehenden Theorien abzugrenzen. Mit den elektrochemischen Potentialen ist auch die Strom-Spannungs-Abhängigkeit in geschlossener Form gegeben. Um zu quantitativen Ergebnissen zu kommen, betrachten wir speziell einen symmetrischen $\mathrm{p}-\mathrm{n}$-Übergang. Wir führen einen Parameter $\alpha$ ein, der von der angelegten Spannung $V$ und der Diffusionslänge $L$ abhängt. Das Produkt $\alpha V$ ist als eine über die Ortskoordinate $x$ gemittelte Aufspaltung der elektrochemischen Potentiale im Übergangsgebiet definiert. Wir bestimmen $\alpha$ in seiner funktionalen Abhängigkeit aus einer Randbedingung, der die elektrochemischen Potentiale genügen und sind damit in der Lage, die berechneten Charakteristiken quantitativ zu diskutieren.

5 O. Madelung, Z. Naturforschg. 12 a, 1020 [1957].

\section{Allgemeiner Formalismus}

\section{a) Schematische Darstellung eines $p-n$-Übergangs}

Für unsere Rechnungen wählen wir ein eindimensionales Modell. Abb. 1 veranschaulicht den $p-n$ Übergang im Gleichgewicht, Abb. 2 gibt die Verhältnisse unter einer äußeren Spannung $V=v \cdot k T / q$ wieder. Wie in den beiden Abbildungen gezeigt

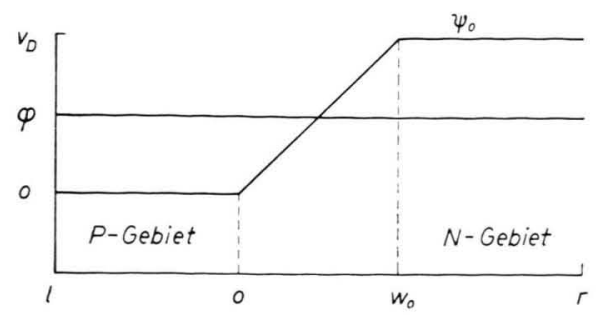

Abb. 1. Der $\mathrm{p}-\mathrm{n}$-Übergang im Gleichgewicht.

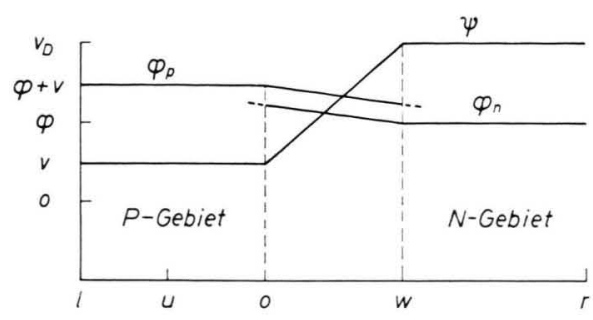

Abb. 2. Der belastete $\mathrm{p}-\mathrm{n}$-Übergang.

wird, geht bei Belastung das elektrostatische Potential $\psi_{0}$ über in $\psi$, die Breite $w_{0}$ des Übergangsgebietes in $w$, und das Fermi-Potential $\varphi$ spaltet auf in die elektrochemischen Potentiale $\varphi_{\mathrm{n}}$ und $\varphi_{\mathrm{p}} . l$ sei die linke, $r$ die rechte Begrenzung des Halbleiters. Die Potentiale messen wir als Vielfache von $k T / q$ und bezeichnen sie in ihrer dimensionslosen Schreibweise als reduzierte Potentiale. Dann ist die Dichte der Elektronen gegeben durch

$$
n=n_{\mathrm{i}} \exp \left(\psi-\varphi_{\mathrm{n}}\right),
$$

die der Löcher durch

$$
p=n_{\mathrm{i}} \exp \left(\varphi_{\mathrm{p}}-\psi\right),
$$

$n_{\mathrm{i}}$ ist die Eigenleitungskonzentration.

Für das P-Gebiet gilt: Die Dichte der Löcher ändert sich bei Belastung praktisch nicht. Dann muß nach (2) $\varphi_{\mathrm{p}}-\psi=\varphi-\psi_{0}$ sein. Da in unserer Darstellung $\psi=\psi_{0}+v$ ist, muß $\varphi_{\mathrm{p}}=\varphi+v$ werden. Die Dichte der Elektronen ist dagegen in einem Gebiet $u \leqq x \leqq 0$ von der Spannung abhängig. Wir wählen $l \mid$ so groß, daß $n(l)$ den Gleichgewichtswert $n_{\mathrm{p}}$ behält. Dann gilt: $\varphi_{\mathrm{n}}(l)=\varphi_{\mathrm{p}}(l)=\varphi+v$. Analoge 
Verhältnisse herrschen im N-Gebiet: Es gilt $\psi=\psi_{0}$, $\varphi_{\mathrm{n}}=\varphi$ und, indem wir $r$ hinreichend groß wählen, $\varphi_{\mathrm{p}}(r)=\varphi_{\mathrm{n}}(r)=\varphi$. In den betrachteten Abbildungen haben wir den Potentialverlauf im Übergangsgebiet linear approximiert. Diese Näherung werden wir unserer Arbeit zugrunde legen.

b) Integration der Differentialgleichungen für die elektrochemischen Potentiale $\varphi_{\mathrm{n}}(x)$ und $\varphi_{\mathrm{p}}(x)$

Die Ausgangsgleichungen zur Berechnung der elektrochemischen Potentiale $\varphi_{\mathrm{n}}$ und $\varphi_{\mathrm{p}}$ lauten:

$$
\begin{aligned}
& j_{\mathrm{n}}=D_{\mathrm{n}} n \varphi_{\mathrm{n}}{ }^{\prime}, \quad j_{\mathrm{n}}{ }^{\prime}=-U ; \\
& j_{\mathrm{p}}=-D_{\mathrm{p}} p \varphi_{\mathrm{p}}{ }^{\prime}, \quad j_{\mathrm{p}}{ }^{\prime}=-U .
\end{aligned}
$$

Der Index $\mathrm{n}$ charakterisiert die Elektronen, der Index $\mathrm{p}$ die Löcher. $j_{\mathrm{n}}, j_{\mathrm{p}}$ sind die Teilchenstromdich-

ten, $D_{\mathrm{n}}$ und $D_{\mathrm{p}}$ die Diffusionskonstanten, $n$ ist die Elektronendichte, $p$ die Löcherdichte. Mit $U$ bezeichnen wir die Rekombinationsquote, die unter stationären Bedingungen für Elektronen und Löcher gleich ist.

Im folgenden berechnen wir ausgehend von den Gln. (3) und (4) das elektrochemische Potential $\varphi_{\mathrm{n}}$. Wir setzen (1) in (3) ein, eliminieren $j_{n}$ aus (3) und (4) und substituieren

$$
y_{\mathrm{n}}=\frac{\mathrm{d}}{\mathrm{d} x} e^{-\varphi_{\mathrm{n}}} .
$$

Dann erhalten wir die Differentialgleichung

$$
y_{\mathrm{n}}{ }^{\prime}+f(x) \cdot y_{\mathrm{n}}=g(x)
$$

mit den Abkürzungen

$$
f(x)=\psi^{\prime}, \quad g(x)=U e^{-\psi /\left(D_{\mathrm{n}} n_{\mathrm{i}}\right) .}
$$

Die Lösung lautet:

$$
y_{\mathrm{n}}(x)=\exp \left\{-\int_{a}^{x} f(t) \mathrm{d} t\right\} \cdot\left[y_{\mathrm{n}}(a)+\int_{a}^{x} g(u) \cdot \exp \left\{\int_{a}^{u} f(t) \mathrm{d} t\right\} \mathrm{d} u\right] .
$$

Durch Integrieren und Logarithmieren von (7) finden wir

$$
\varphi_{\mathrm{n}}(x)=\varphi_{\mathrm{n}}(b)-\ln \left[1+\exp \left\{\varphi_{\mathrm{n}}(b)\right\} \int_{b}^{x} y_{\mathrm{n}}(\xi) \mathrm{d} \xi\right] .
$$

Drücken wir die in (10) auftretende Integrationskonstante $y_{\mathrm{n}}(a)$ durch Größen mit unmittelbarer physikalischer Bedeutung aus und ersetzen $f(x)$ und $g(x)$ wieder durch die ursprünglichen Funktionen, so folgt:

$$
y_{\mathrm{n}}(x)=-e^{-\psi(x)} /\left(D_{\mathrm{n}} n_{\mathrm{i}}\right) \cdot\left[j_{\mathrm{n}}(a)-\int_{a}^{x} U(t) \mathrm{d} t\right] .
$$

Die Berechnung von $\varphi_{\mathrm{p}}(x)$, ausgehend von den Gln. (5) und (6), läuft vollkommen analog und liefert

$$
\begin{aligned}
& \varphi_{\mathrm{p}}(x)=\varphi_{\mathrm{p}}(\bar{b})+\ln \left[1+\exp \left\{-\varphi_{\mathrm{p}}(\bar{b})\right\} \int_{\bar{b}}^{x} y_{\mathrm{p}}(\xi) \mathrm{d} \xi\right], \\
& y_{\mathrm{p}}(x)=-e^{\psi(x)} /\left(D_{\mathrm{p}} n_{\mathrm{i}}\right) \cdot\left[j_{\mathrm{p}}(\bar{a})-\int_{\bar{a}}^{x} U(t) \mathrm{d} t\right] .
\end{aligned}
$$

Die Gln. (11) bis (14) gelten ganz allgemein. Sie enthalten weder vereinfachende Ansätze noch spezielle Annahmen.

$$
\text { c) } \varphi_{\mathrm{n}}(x) \text { im P-Gebiet, } \varphi_{\mathrm{p}}(x) \text { im N-Gebiet }
$$

Wir ersetzen in (11) $b$ und in (12) $a$ durch die linke Begrenzung des Halbleiters, deren Koordinatenwert wir $l$ genannt haben. $|l|$ ist nach Voraussetzung so groß, daß $j_{\mathrm{n}}(l)=0$ wird. Dann folgt aus $(12)$ :

$$
y_{\mathrm{n}}(x)=e^{-\psi(x)} /\left(D_{\mathrm{n}} n_{\mathrm{i}}\right) \cdot \int_{i}^{x} U(t) \mathrm{d} t .
$$

Im P-Gebiet ist die Rekombinationsquote $U(x)$ proportional zur Abweichung der Elektronendichte von ihrem Gleichgewichtswert, vorausgesetzt, daß diese klein gegen die Löcherdichte bleibt:

$$
U(x)=\left(n(x)-n_{\mathrm{p}}\right) / \tau_{\mathrm{n}}, \quad x \leqq 0 ;
$$

$\tau_{\mathrm{n}}$ ist die Lebensdauer der Elektronen im P-Gebiet. 
$n(x)-n_{\mathrm{p}}$ berechnen wir aus den Gln. (3) und (4), indem wir den Feldstrom neben dem Diffusionsstrom vernachlässigen. Damit haben wir $U(x)$ und finden:

$$
\begin{aligned}
& y_{\mathrm{n}}(x)=e^{-\psi(x)} /\left(L_{\mathrm{n}} n_{\mathrm{i}}\right)\left\{n(0)-n_{\mathrm{p}}\right\} \exp \left\{x / L_{\mathrm{n}}\right\}, \\
& \varphi_{\mathrm{n}}(x)=\varphi_{\mathrm{n}}(l)-\ln \left\{1+\left[\frac{n(0)}{n_{\mathrm{p}}}-1\right] \cdot \exp \left\{x / L_{\mathrm{n}}\right\}\right\} \quad \text { für } \quad x \leqq 0 \quad \text { (P-Gebiet) } .
\end{aligned}
$$

Wir haben $D_{\mathrm{n}} \tau_{\mathrm{n}}=L_{\mathrm{n}}{ }^{2}$ gesetzt. $L_{\mathrm{n}}$ ist die Diffusionslänge der Elektronen im P-Gebiet. Bei den vorkommenden Integrationen haben wir die untere Integrationsgrenze $l$ durch $-\infty$ ersetzt. Außerdem haben wir das im P-Gebiet nahezu konstante Potential $\psi(x)$ durch $\psi(l)$ approximiert.

In vollkommen analoger Weise berechnet sich $\varphi_{\mathrm{p}}(x)$ im N-Gebiet. Wir erhalten das Ergebnis:

$$
\varphi_{\mathrm{p}}(x)=\varphi_{\mathrm{p}}(\boldsymbol{r})+\ln \left\{1+\left[\frac{p(w)}{p_{\mathrm{n}}}-1\right] \cdot \exp \left\{-(x-w) / L_{\mathrm{p}}\right\} ; \text { für } x \geqq w \quad \text { (N-Gebiet) } ;\right.
$$

$L_{\mathrm{p}}$ ist die Diffusionslänge der Löcher im N-Gebiet.

$$
\text { d) } \varphi_{\mathrm{n}}(x) \text { im N-Gebiet, } \varphi_{\mathrm{p}}(x) \text { im P-Gebiet }
$$

Um das elektrochemische Potential $\varphi_{\mathrm{n}}(x)$ im N-Gebiet zu berechnen, ersetzen wir in (11) $b$ und in (12) $a$ durch den Koordinatenwert $r$ der rechten Begrenzung des Halbleiters. $r$ ist entsprechend $l$ so groß vorausgesetzt, daß der Strom an dieser Stelle nur von den Elektronen getragen wird. Wir schreiben $j_{\mathrm{n}}(r)=-j$, dann gilt nach $(12)$ :

$$
y_{\mathrm{n}}(x)=e^{-\psi(x)} /\left(D_{\mathrm{n}} n_{\mathrm{i}}\right) \cdot\left\{j+\int_{r}^{x} U(t) \mathrm{d} t\right\} .
$$

Im N-Gebiet ist die Rekombinationsquote $U(x)$ proportional zur Abweichung der Löcherdichte von ihrem Gleichgewichtswert, vorausgesetzt, daß diese klein gegen die Elektronendichte bleibt:

$$
U(x)=\left(p(x)-p_{\mathrm{n}}\right) / \tau_{\mathrm{p}}, \quad x \geqq w ;
$$

$\tau_{\mathrm{p}}$ ist die Lebensdauer der Löcher im N-Gebiet.

In Analogie zur Berechnung von $\varphi_{\mathrm{n}}(x)$ im P-Gebiet folgt für das N-Gebiet:

$$
\begin{gathered}
\varphi_{\mathrm{n}}(x)=\varphi_{\mathrm{n}}(r)-\ln \left\{1-\left(1 / D_{\mathrm{n}} n_{\mathrm{n}}\right) \cdot\left[j(r-x)-D_{\mathrm{p}}\left(p(w)-p_{\mathrm{n}}\right) \cdot \exp \left\{-(x-w) / L_{\mathrm{p}}\right\}\right]\right\} \\
\text { für } x \geqq w \quad(\mathrm{~N} \text {-Gebiet }) .
\end{gathered}
$$

Bei der Durchführung der Rechnung haben wir $\psi(x)$ durch $\psi(r)$ und bei den vorkommenden Integrationen über $\exp \left\{-(x-w) / L_{\mathrm{p}}\right\}$ die Integrationsgrenze $r$ durch $+\infty$ ersetzt. Das sind gute Näherungen.

Für $\varphi_{\mathrm{p}}(x)$ im P-Gebiet ergibt sich entsprechend:

$\varphi_{\mathrm{p}}(x)=\varphi_{\mathrm{p}}(l)+\ln \left\{1-\left(1 / D_{\mathrm{p}} p_{\mathrm{p}}\right)\left[j(x-l)-D_{\mathrm{n}}\left(n(0)-n_{\mathrm{p}}\right) \exp \left\{x / L_{\mathrm{n}}\right\}\right]\right\}$ für $\quad x \leqq 0$ (P-Gebiet).

\section{e) $\varphi_{\mathrm{n}}(x)$ im Übergangsgebiet eines symmetrischen $\mathrm{p}-\mathrm{n}$-Übergangs}

Die bisherigen Rechnungen konnten wir unter den üblichen Annahmen durchführen, daß die Rekombinationsquote in den Homogengebieten proportional zur Abweichung der Minoritätsträgerdichte von ihrem Gleichgewichtswert ist und daß eben dort der Feldstrom der Minoritätsträger neben ihrem Diffusionsstrom vernachlässigt werden kann. Für alle nun folgenden Überlegungen müssen wir weitere Vereinfachungen vornehmen. Wir beschränken uns auf den symmetrischen $\mathrm{p}-\mathrm{n}$-Übergang und machen Approximationen, auf die wir an gegebener Stelle hinweisen. Da die vorausgesetzte Symmetrie des $\mathrm{p}$-n-Übergangs auch im Verlauf der elektrochemischen Potentiale zum Ausdruck kommt, genügt es, die Funktion $\varphi_{\mathrm{n}}(x)$ zu berechnen. $\varphi_{\mathrm{p}}(x)$ ist dann gleichzeitig gegeben.

Ausgehend von (11) können wir schreiben:

$$
\begin{aligned}
\varphi_{\mathrm{n}}(x) & =\varphi_{\mathrm{n}}(l) \\
- & \ln \left\{1+e^{\varphi_{\mathrm{n}}(l)} \int_{i}^{0} y_{\mathrm{n}}(\xi) \mathrm{d} \xi+e^{\varphi_{\mathrm{n}}(l)} \int_{0}^{x} y_{\mathrm{n}}(\xi) \mathrm{d} \xi\right\} .
\end{aligned}
$$

Der zweite Summand im Argument des Logarithmus läßt sich nach (17) bestimmen. Es bleibt die Auf- 
gabe, den dritten Summanden zu berechnen. Nach (12) gilt:

$$
y_{\mathrm{n}}(x)=-\frac{e^{-\psi(x)}}{D_{\mathrm{n}} n_{\mathrm{i}}}\left\{j_{\mathrm{n}}(0)-\int_{0}^{x} U(t) \mathrm{d} t\right\} .
$$

Wir machen die Annahme, daß die Rekombination über Rekombinationszentren erfolge, die energetisch zwischen dem Valenz- und Leitungsband gelegen sind. Für diesen Fall haben Shockley und ReAD ${ }^{6}$ den Ausdruck hergeleitet:

$$
U=\frac{p n-n_{\mathrm{i}}^{2}}{\left(n+n_{1}\right) \tau_{\mathrm{p}}+\left(p+p_{1}\right) \tau_{\mathrm{n}}} ;
$$

$n_{1}$ ist die Dichte der Elektronen im Leitungsband, wenn das Fermi-Niveau mit dem Niveau der Rekombinationszentren zusammenfällt. $p_{1}$ ist die entsprechende Dichte der Löcher im Valenzband.

Wegen der Symmetrie des Problems können wir setzen:

$$
\begin{gathered}
\tau_{\mathrm{n}}=\tau_{\mathrm{p}}=\tau, \quad D_{\mathrm{n}}=D_{\mathrm{p}}=D, \\
n(0)=n_{\mathrm{p}} e^{\alpha v} ; \quad n(w)=n_{\mathrm{n}} ; \\
p(w)=p_{\mathrm{n}} e^{\alpha v} ; \quad p(0)=p_{\mathrm{p}} ; \\
n(x)=p(w-x) ;
\end{gathered}
$$

$\alpha$ ist ein später zu bestimmender Parameter.

Für die folgenden Rechnungen machen wir die Approximation:

$$
n p=n_{\mathrm{i}}{ }^{2} e^{\varphi_{\mathrm{p}}-\varphi_{\mathrm{n}}}=n(0) p(0)=n(w) p(w)=n_{\mathrm{i}}{ }^{2} e^{\alpha v} .
$$

Sie beinhaltet, daß wir uns nicht auf konstante elektrochemische Potentiale im Übergangsgebiet beschränken, sondern auch Fälle mit $\left|\varphi_{\mathrm{p}}-\varphi_{\mathrm{n}}\right|<|v|$ erfassen wollen. Die Näherung besteht darin, daß wir $\alpha$ und damit $\varphi_{\mathrm{p}}-\varphi_{\mathrm{n}}$ als unabhängig von der Ortskoordinate ansehen. Legen wir außerdem die energetische Lage der Rekombinationszentren auf die Mitte der verbotenen Zone fest, so gilt:

$$
n_{1}=p_{1}=n_{\mathrm{i}},
$$

und wir erhalten als Rekombinationsquote:

$$
\quad U=\left(n_{\mathrm{i}}^{2} / \tau\right)\left(e^{\alpha v}-1\right) \frac{1}{n+p+2 n_{\mathrm{i}}} \text {. }
$$

Bernard ${ }^{2}$, Sah u. a. ${ }^{3}$ und Chevychelov ${ }^{4}$ betrachten Fälle, in denen $\alpha=1$ ist. Wir werden $\alpha$ in Abhängigkeit von der angelegten Spannung $v$ und der Diffusionslänge $L$ bestimmen, um so den Gültigkeitsbereich dieser Theorien abgrenzen zu können. $\alpha=1$ bedeutet, daß die elektrochemischen Potentiale im Übergangsgebiet konstant sind. Bei hinreichend kleinen Diffusionslängen ist das aber nicht der Fall. Kleine Diffusionslänge heißt große Rekombination. Unser Ziel ist es, zu untersuchen, wie sich die Strom-Spannungs-Charakteristik eines symmetrischen $\mathrm{p}$-n-Übergangs mit zunehmender Rekombination ändert. Insbesondere interessieren die Verhältnisse bei Übergängen, die durch die obengenannten Theorien nicht mehr beschrieben werden. Der Grenzfall $L=0$ ist, wie in der Einleitung erwähnt wurde, von $\mathrm{M}_{\mathrm{ADELUNG}}{ }^{5}$ behandelt.

Nach (30) können wir schreiben:

$$
\int_{0}^{x} U(t) \mathrm{d} t=\frac{n_{\mathrm{i}}^{2}}{\tau} \cdot\left(e^{\alpha v}-1\right) \int_{0}^{x} \frac{\mathrm{d} t}{n(t)+p(t)+2 n_{\mathrm{i}}} .
$$

Wählen wir $n$ als neue Integrationsvariable und setzen gemäß einer linearen Approximation der Potentiale

so erhalten wir mit (28)

$$
\left(\psi-\varphi_{\mathrm{n}}\right)^{\prime}=\gamma=\frac{v_{\mathrm{D}}-\alpha v}{w}, \quad \psi^{\prime}=\varkappa=\frac{v_{\mathrm{D}}-v}{w},
$$

$$
\int_{0}^{x} U(t) \mathrm{d} t=\frac{n_{\mathrm{i}} \sqrt{e^{\alpha v-1}-1}}{\gamma \tau}\left\{\arctan \frac{\left(n_{\mathrm{n}} / n_{\mathrm{i}}\right)+1}{\sqrt{e^{\alpha v}-1}}-\arctan \frac{n(w-x) / n_{\mathrm{i}}+1}{\sqrt{e^{\alpha v-1}}}\right\} .
$$

Einsetzen von (34) in (25) und (25) in (24) liefert das gesuchte Ergebnis:

$$
\begin{aligned}
& \varphi_{\mathrm{n}}(x)=\varphi_{\mathrm{n}}(l)-\ln \left\{e^{\alpha v}+\left(\frac{w}{L}\right)^{2} \cdot T(x)+\frac{1-\exp \left\{-\left(v_{\mathrm{D}}-v\right) x / w\right\}}{v_{\mathrm{D}}-v}\right. \\
& \cdot\left[\left(\frac{w}{L}\right)^{2} \frac{n_{\mathrm{i}}}{n_{\mathrm{p}}} \frac{\sqrt{e^{\alpha v-1}}}{v_{\mathrm{D}}-\alpha v} \cdot \arctan \frac{\left(n_{\mathrm{n}} / n_{\mathrm{i}}\right)+1}{\left.\left.\sqrt{e^{\alpha v}-1}+\frac{w}{L} \cdot\left(e^{\alpha v}-1\right)\right]\right\}}\right. \\
& T(x)=-\frac{1}{w} \frac{n_{\mathrm{i}}}{n_{\mathrm{p}}} \frac{\sqrt{e^{\alpha v-1}}}{v_{\mathrm{D}}-\alpha v} \int_{0}^{x} e^{-x \xi \cdot \arctan \frac{n(w-\xi) / n_{\mathrm{i}}+1}{\sqrt{e^{\alpha v-1}}} \cdot \mathrm{d} \xi} .
\end{aligned}
$$

${ }^{6}$ W. Shockley u. W. T. Read, Phys. Rev. 87, 835 [1952]. 
(35) beschreibt den Verlauf des elektrochemischen Potentials $\varphi_{\mathrm{n}}(x)$ im Übergangsgebiet. Um $\int_{0}^{x} U(t) \mathrm{d} t$ zu berechnen, haben wir das elektrostatische Potential und die elektrochemischen Potentiale linear angesetzt. Diese Näherung ist durch die weitgehende Übereinstimmung der Ergebnisse von Bernard ${ }^{2}$ und $\mathrm{S}_{\mathrm{AH}}$ u. a. ${ }^{3}$ mit denen Chevychelovs ${ }^{4}$ gerechtfertigt. Der Parameter $\alpha$ in Abhängigkeit von $v$ und $L$ ist durch die Randbedingung

$$
\varphi_{\mathrm{n}}(w)=\varphi_{\mathrm{n}}(r)
$$

zu bestimmen. Die Breite $w$ des Übergangsgebietes wird im folgenden Kapitel als Funktion von $v$ und $\alpha$ berechnet. (36) gilt nicht exakt, der Fehler läßt sich aber im Einzelfall nach Gl. (22) abschätzen. Er erweist sich in unserem numerischen Beispiel in weiten Grenzen als vernachlässigbar klein.

f) Die Breite $w$ des Übergangsgebietes in Abhängigkeit von der angelegten Spannung $v$ und dem

\section{Parameter a}

In Gl. (35) ist das elektrochemische Potential $\varphi_{\mathrm{n}}(x)$ für das Übergangsgebiet gegeben. Parameter sind $\alpha, v, L$ und $w$. Wie die folgende Rechnung ergibt, ist $w$ seinerseits eine Funktion von $v$ und $\alpha$. Diese funktionale Abhängigkeit müssen wir kennen, um aus Bedingung (36) $\alpha$ als Funktion von $v$ und $L$ berechnen zu können.

Das elektrostatische Potential $\psi$, gemessen in der Einheit $k T / q$ genügt der Differentialgleichung:

$$
\frac{\mathrm{d}^{2} \psi}{\mathrm{d} x^{2}}=-\frac{q}{k T} \frac{q}{\varepsilon \varepsilon_{0}}\left\{p-n+N_{\mathrm{D}}-N_{\mathrm{A}}\right\} .
$$

$N_{\mathrm{D}}$ ist die Dichte der Donatoren, $N_{\mathrm{A}}$ die der Akzeptoren, $\varepsilon$ ist die relative Dielektrizitätskonstante, $\varepsilon_{0}$ die Influenzkonstante. Die Elektronendichte $n$ und die Löcherdichte $p$ sind durch die Relationen (1) und (2) gegeben.

Wir berechnen unter Verwendung der bereits benutzten linearen Potentialansätze:

$$
p-n=-2 n(0) e^{\gamma w / 2} \sinh \gamma(x-w / 2) .
$$

Beschränken wir uns wieder auf den symmetrischen $\mathrm{p}-\mathrm{n}$-Übergang, dann genügt es, das Potential $\psi$ im Gebiet $0 \leqq x \leqq w / 2$ zu betrachten. Außerdem sei der Übergang abrupt, so daß gilt:

$$
N_{\mathrm{D}}=0 \text { und } N_{\mathrm{A}}=n_{\mathrm{n}} \text { für } x \leqq w / 2 .
$$

Mit den Randbedingungen

$$
\psi^{\prime}(0)=0, \quad \psi(0)=v
$$

erhalten wir durch Integration der Diff.-Gl. (37) :

$$
\begin{aligned}
\psi(x)=\frac{1}{2} f x^{2}+g & \left\{\frac{1}{\gamma^{2}} \sinh \gamma\left(x-\frac{w}{2}\right)\right. \\
- & \left.-\frac{x}{\gamma} \cosh \gamma \frac{w}{2}+\frac{1}{\gamma^{2}} \sinh \gamma \frac{w}{2}\right\}+v \\
\text { für } 0 \leqq x \leqq w / 2 & \\
\text { mit } \quad f & =\frac{q}{k T \quad \frac{q}{\varepsilon \varepsilon_{0}} n_{\mathrm{n}},} \\
g & =\frac{q}{k T} \quad q \quad 2 \varepsilon_{0} n(0) e^{\gamma w / 2} \\
\gamma & =\frac{v_{\mathrm{D}}-\alpha v}{w}=\frac{b}{w}, \\
n(0) & =n_{\mathrm{p}} e^{\alpha v} .
\end{aligned}
$$

Die Bedingung

$$
\psi(w / 2)=v+\frac{1}{2}\left(v_{\mathrm{D}}-v\right)
$$

liefert $w$ in Abhängigkeit von $v$ und $\alpha$ :

$v_{\mathrm{D}}-v=w^{2}\left\{\frac{1}{4} f-\frac{1}{b} g \cosh \frac{b}{2}+\frac{2}{b^{2}} g \sinh \frac{b}{2}\right\}$.

Damit haben wir $w$ als Funktion von $v$ und $\alpha$ berechnet. Der Klammerausdruck hängt nur von $\alpha v$ ab, wie wir bei Einsetzen der Abkürzungen (43) bis (46) erkennen. Setzen wir $w=w(v, \alpha)$ in (35) ein, dann können wir nach (36) $\alpha=\alpha(v, L)$ bestimmen.

\section{g) Die Gesamtstromdichte $i$}

Zur Berechnung der Gesamtstromdichte $i$ greifen wir zurück auf die Gln. (3), (5) und erhalten:

$$
i=-q j_{\mathrm{n}}+q j_{\mathrm{p}}=q\left\{-D_{\mathrm{n}} n \varphi_{\mathrm{n}}{ }^{\prime}-D_{\mathrm{p}} p \varphi_{\mathrm{p}}{ }^{\prime}\right\} .
$$

Bilden wir nach (11) und (13) die Ableitungerı $\varphi_{\mathrm{n}}{ }^{\prime}$ und $\varphi_{\mathrm{p}}{ }^{\prime}$, so fo!gt

$$
i=q\left\{-j_{\mathrm{n}}(0)+j_{\mathrm{p}}(w)+\int_{0}^{w} U(t) \mathrm{d} t\right\} .
$$

Dieser Ausdruck für die Stromdichte $i$ ist von allgemeiner Gültigkeit. Die beiden ersten Summanden entsprechen dem SноскLEyschen Grenzfall geringer Rekombination ${ }^{1}$; mit dem Integral über $U$ wird der Beitrag der Rekombination im Übergangsgebiet hinzugefügt.

Beschränken wir uns wieder auf den symmetrischen $\mathrm{p}-\mathrm{n}$-Übergang, so gelten die Beziehungen:

$$
\begin{gathered}
-j_{\mathrm{n}}(0)=j_{\mathrm{p}}(w), \\
n(0)=n_{\mathrm{p}} e^{x x}, \\
D_{\mathrm{n}}=D_{\mathrm{p}}=D, \quad L_{\mathrm{n}}=L_{\mathrm{p}}=L .
\end{gathered}
$$


$j_{\mathrm{n}}(0)$ berechnet sich aus (3) und (4) bei Vernachlässigung des Feldstromes. Wir erhalten das Ergebnis:

$$
i=q\left\{2 \frac{D}{L} n_{\mathrm{p}}\left(e^{\alpha v}-1\right)+\int_{0}^{w} U(t) \mathrm{d} t\right\} .
$$

Das Integral über die Rekombinationsquote $U$ ist mit der Approximation linearer Potentiale in (34) gegeben. Haben wir nach (36) zusammen mit (48) den Parameter $\alpha=\alpha(v, L)$ bestimmt, so können wir aus (54) die Strom-Spannungs-Charakteristik eines symmetrischen $\mathrm{p}-\mathrm{n}$-Übergangs $\mathrm{zu}$ vorgegebener Diffusionslänge $L$ ermitteln. Die Breite $w$ des Übergangsgebietes ist Gl. (48) zu entnehmen. Wir werden im folgenden diese allgemeinen Überlegungen auf ein spezielles Beispiel anwenden.

\section{Numerische Behandlung eines speziellen p-n-Übergangs, Anwendung des allgemeinen Formalismus}

Wir haben den allgemeinen Formalismus bereits insofern spezialisiert, als wir uns bei der Berechnung der elektrochemischen Potentiale und des elektrostatischen Potentials im Übergangsgebiet auf den abrupten symmetrischen $\mathrm{p}-\mathrm{n}$-Übergang beschränkt haben. Unserer numerischen Betrachtung legen wir folgenden Zahlenwert zugrunde:

$$
n_{\mathrm{n}} / n_{\mathrm{i}}=n_{\mathrm{i}} / n_{\mathrm{p}}=10^{5} .
$$

Die reduzierte Diffusionsspannung $v_{\mathrm{D}}$ berechnet sich dann zu

$$
v_{\mathrm{D}}=\ln \left(n_{\mathrm{n}} / n_{\mathrm{p}}\right)=\ln 10^{10}=23,0259,
$$

was gleichbedeutend ist mit

$$
V_{\mathrm{D}}=0,60 \text { Volt. }
$$

Diese Zahlenangaben genügen vorerst.

\section{a) Ermittlung des Parameters $\alpha$ in Abhängigkeit von $v$ für vorgegebenes $L$}

Wir lösen die gestellte Aufgabe, indem wir zunächst den Quotienten $w / L$ als Funktion von $\alpha$ und $v$ berechnen, dann $w_{0} / w$ ermitteln und durch Produktbildung $w_{0} / L$ erhalten. $w_{0}$ ist die Breite des Übergangsgebietes im unbelasteten Fall. Zur Durchführung dieses Programms setzen wir in (35) $x=w$. Dann liefert die Randbedingung (36) eine quadratische Bestimmungsgleichung für den Quotienten $w / L$. Da $w / L$ positiv sein muß, ist die Lösung eindeutig. Den Quotienten $w_{0} / w$ berechnen wir nach
Gl. (48); sie ist ebenfalls eine quadratische Bestimmungsgleichung. Setzen wir

$$
w_{0} / L=1 / \lambda,
$$

so ist $\lambda$ die Diffusionslänge, gemessen in der Einheit $w_{0}$. Wählen wir je eine Folge von $\alpha$-Werten und eine Folge von $v$-Werten, so können wir $\lambda$ als Funktion von $v$ für die verschiedenen $\alpha$ darstellen. Damit kennen wir auch $\alpha$ als Funktion von $v$ für festes $\lambda$.

Wir wollen uns in unserem Beispiel auf positive Spannungen $v$ beschränken, den $\mathrm{p}-\mathrm{n}$-Übergang also bei Flußbelastung untersuchen. Da unsere Theorie auf der Voraussetzung aufbaut, daß die Rekombinationsquote $U$ in P- und N-Gebiet proportional zur Abweichung der Minoritätsträgerdichten von ihrem Gleichgewichtswert sei, kann sie nur gelten, wenn diese Forderung erfüllt ist. Sie ist erfüllt, solange die Minoritätsträgerdichte klein bleibt gegen die Majoritätsträgerdichte, was gleichbedeutend ist damit, daß die angelegte Spannung $v$ eine obere Grenze nicht überschreitet. Diese Grenze ist natürlich nicht scharf definiert. Nehmen wir an, daß

sei, so ergibt sich

$$
n(0)=n_{\mathrm{p}} e^{a v} \leqq n_{\mathrm{n}} / 100
$$

$$
v \leqq 18,4 / \alpha, \quad \alpha \leqq 1 .
$$

Wir können also sagen, daß unsere Theorie bis zu einer Spannung $V=18,4(k T / q) \simeq 0,5$ Volt gelten muß.

Der Parameter $\alpha$ ist in dem Bereich $0 \leqq \alpha \leqq 1$ zu wählen. $\alpha=1$ bedeutet, daß die elektrochemischen

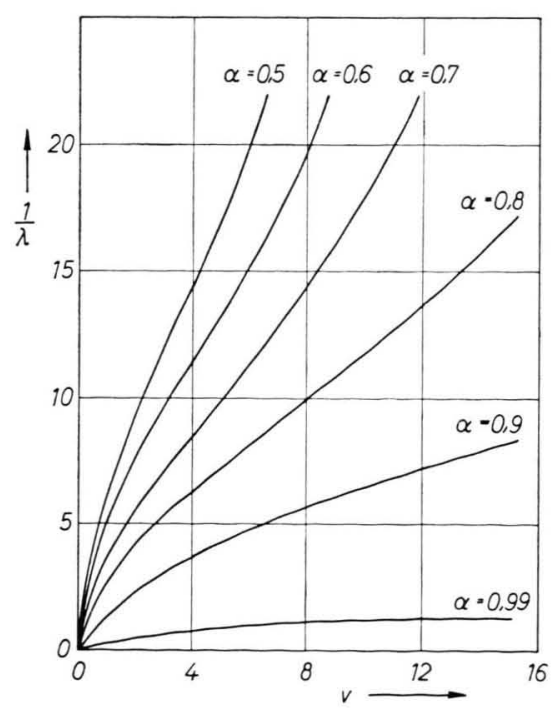

Abb. 3. $1 / \lambda$ als Funktion von $v$ (Parameter $\alpha)$. 


\begin{tabular}{|c|c|c|c|c|c|c|c|}
\hline${ }_{\alpha} v$ & 3 & 6 & 9 & 12 & 15 & 18 & 21 \\
\hline $\begin{array}{l}0,2 \\
0,3 \\
0,4 \\
0,5 \\
0,6 \\
0,7 \\
0,8 \\
0,9 \\
0,99\end{array}$ & $\begin{array}{c}23,9 \\
18,6 \\
15,5 \\
12,0 \\
9,75 \\
7,14 \\
5,32 \\
3,11 \\
0,60\end{array}$ & $\begin{array}{c}47,6 \\
33,9 \\
25,1 \\
20,0 \\
15,2 \\
11,3 \\
8,07 \\
4,75 \\
1,00\end{array}$ & $\begin{array}{c}95,4 \\
63,0 \\
44,6 \\
32,1 \\
23,3 \\
16,1 \\
10,8 \\
6,06 \\
1,15\end{array}$ & $\begin{array}{c}203 \\
116 \\
76,4 \\
51,2 \\
34,2 \\
22,4 \\
13,7 \\
7,26 \\
1,20\end{array}$ & $\begin{array}{c}444 \\
209 \\
128 \\
80,2 \\
49,4 \\
29,7 \\
16,9 \\
8,24 \\
1,30\end{array}$ & $\begin{array}{c}1030 \\
376 \\
214 \\
124 \\
70,2 \\
38,8 \\
20,2 \\
9,00\end{array}$ & $\begin{array}{r}2550 \\
762 \\
401 \\
214 \\
111 \\
55,7 \\
26,0 \\
10,2\end{array}$ \\
\hline
\end{tabular}

Tab. 1. $1 / \lambda$ in Abhängigkeit von $v$ und $\alpha$.

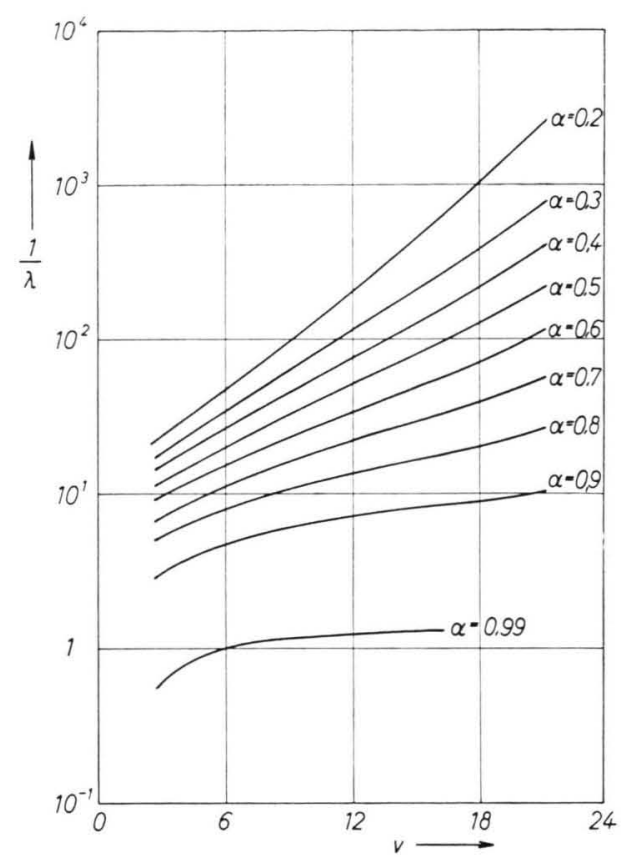

Abb. 4. $1 / \lambda$ als Funktion von $v$ in logarithmischer Darstellung (Parameter $\alpha$ ).

Potentiale im Übergangsgebiet konstant bleiben und ihre Aufspaltung $v$ beträgt. Dieser Fall wurde von Bernard ${ }^{2}$ und $\mathrm{S}_{\mathrm{AH}}$ u. a. ${ }^{3}$ behandelt und entspricht geringer Rekombination im Übergangsgebiet. CHEvychelovs ${ }^{4}$ Theorie hat noch spezielleren Charakter. Nimmt die Größe der Rekombination zu, so nimmt $\alpha$ ab, und im Grenzfall unendlich hoher Rekombination ${ }^{5}$ muß $\alpha$ verschwinden.

Die berechneten reziproken Diffusionslängen $1 / \lambda$ zu vorgegebenen $v$ - und $\alpha$-Werten stellen wir in Tab. 1 zusammen; wir veranschaulichen $1 / \lambda$ in $A b$ hängigkeit von $v$ für verschiedene Parameterwerte $\alpha$ in den Abb. 3 und 4. Aus diesen Abbildungen läßt sich $\alpha$ als Funktion von $v$ für vorgegebenes $\lambda$ entnehmen.

\section{b) Strom-Spannungs-Charakteristiken}

Wir haben die Stromdichte $i$ als Funktion von $v$ in Gl. (54) angegeben. Das Integral über die Rekombinationsquote $U$ ist in (34) berechnet. Setzen wir (34) mit $x=w$ in (54) ein, so erhalten wir

$$
i=i_{0}(d+r)
$$

mit den Abkürzungen:

$$
\begin{aligned}
& i_{0}=\left(2 q n_{\mathrm{p}} D / w_{0}\right) \cdot 10^{8}, \\
& d=(1 / \lambda)\left(e^{\alpha v}-1\right) \cdot 10^{-8}, \\
& r=\frac{1}{\lambda^{2}} \frac{w}{2 w_{0}} \frac{\sqrt{e^{\alpha v}-1}}{v_{\mathrm{D}}-\alpha v} \\
& \cdot\left\{\arctan \frac{\left(n_{\mathrm{n}} / n_{\mathrm{i}}\right)+1}{\left.\sqrt{e^{\alpha v}-1}-\arctan \frac{\left(n_{\mathrm{p}} / n_{\mathrm{i}}\right) e^{\alpha v}+1}{\sqrt{e^{\alpha v}-1}}\right\} \cdot 10^{-3} .}\right.
\end{aligned}
$$

$i_{0}$ ist eine geeignet gewählte Stromdichteneinheit. $d$ ist der Diffusionsanteil, $r$ der Rekombinationsanteil des Stromes in der Einheit $i_{0}$.

Fügen wir zu dem Zahlenwert (55) den Wert

$$
n_{\mathrm{p}}=10^{5} \mathrm{~cm}^{-3}
$$

hinzu, so berechnen wir für Silicium mit $\varepsilon=11,7^{7}$ und $k T / q=25,9 \cdot 10^{-3}$ Volt nach (48)

$$
w_{0}=1,4 \cdot 10^{-4} \mathrm{~cm}
$$

und mit $D=20 \mathrm{~cm}^{2} / \mathrm{s}$ nach $(61)$

$$
i_{0}=0,5 \mathrm{~A} / \mathrm{cm}^{2} \text {. }
$$

Unser Ziel ist es, die Stromdichte $i$ in Abhängigkeit von $v$ für verschiedene Werte des Parameters $\lambda$ zu ermitteln. Betrachten wir (61), so sehen wir, daß $v$ und $\alpha$ nie allein, sondern stets als Produkt auftreten. Es ist daher sinnvoll, zunächst $\alpha v$ als Funktion von $v$ zu bestimmen. Ausgehend von den Abb. 3 und 4 erhalten wir die in Tab. 2 zusammengestellten Werte. Die Veranschaulichung des Produktes $\alpha v$ in Abhängigkeit von $v$ finden wir in Abb. 5. $\lambda$ ist Parameter.

7 W. C. Dunlap u. R. L. Watters, Phys. Rev. 92, 1396 [1953]. 


\begin{tabular}{|r|r|r|r|r|}
\hline \multicolumn{1}{r|}{$\lambda$} & $\geq 1$ & $\frac{1}{9}$ & $\frac{1}{27}$ & $\frac{1}{81}$ \\
\hline 2,0 & 2,0 & 1,05 & & \\
3,0 & 3,0 & 1,86 & & \\
4,5 & 4,5 & 3,21 & 1,20 & \\
6,0 & 6,0 & 4,63 & 2,24 & \\
7,5 & 7,5 & 6,08 & 3,56 & \\
9,0 & 9,0 & 7,54 & 5,00 & 2,12 \\
10,5 & 10,5 & 9,03 & 6,44 & 3,31 \\
12,0 & 12,0 & 10,48 & 7,92 & 4,56 \\
15,0 & 15,0 & 13,38 & 10,80 & 7,50 \\
18,0 & 18,0 & 16,20 & 13,64 & 10,35 \\
\hline
\end{tabular}

Tab. 2. $\alpha v$ in Abhängigkeit von $v$ und $\lambda$.

\begin{tabular}{|c|c|c|c|c|c|}
\hline$\lambda$ & $0,5 \cdot 10^{5}$ & 27 & 3 & $\frac{1}{3}$ & $\frac{1}{27}$ \\
\hline$v(\lambda)$ & 11 & 6 & 4 & 2 & 0 \\
\hline
\end{tabular}

Tab. 3. $\nu$ in Abhängigkeit von $\lambda$.

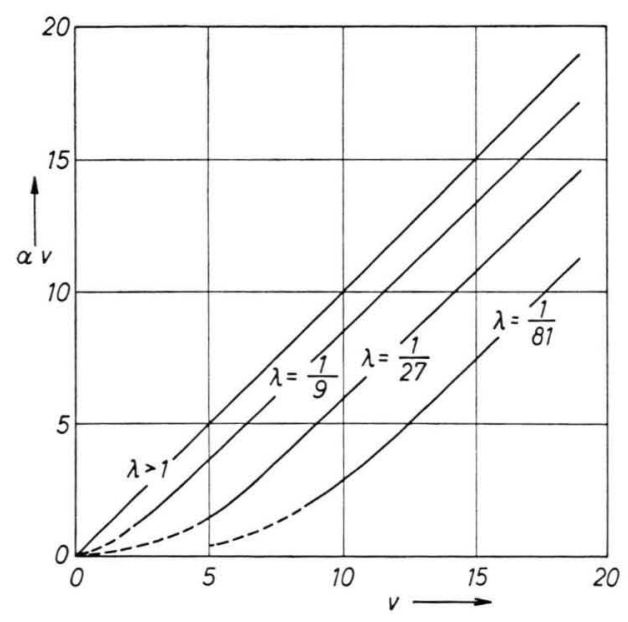

Abb. 5. $a v$ als Funktion von $v$ (Parameter $\lambda$ ).

Nach Gl. (61) können wir nun $i$ bei fest vorgegebenem $\lambda$ für eine Folge von $\alpha v$-Werten berechnen. Aus Kurven entsprechend Abb. 5 finden wir die zugehörigen Spannungen $v$ und sind somit in der Lage, die gesuchten Strom-Spannungs-Charakteristiken darzustellen. Das geschieht in den Abb. 6 und 7 für die Diffusionslängen

$$
\lambda=0,5 \cdot 10^{5} ; 27 ; 3 ; \frac{1}{3} ; \frac{1}{27} .
$$

In Abb. 7 ist zu beachten, daß $\left(i / i_{0}\right) \cdot 10^{-\nu(\lambda)}$ als Ordinate aufgetragen ist, wobei der Exponent $v$ gemäß Tab. 3 von $\lambda$ abhängt. Für die nachfolgende Diskussion geben wir in den drei Tabellen 4, 5 und 6 für

$$
\lambda=0,5 \cdot 10^{5} ; \quad \lambda=27 ; \quad \lambda=\frac{1}{27} ;
$$

die zu vorgegebenen Spannungen $v$ gehörigen Stromdichten $d, r$ und $i / i_{0}=d+r$.

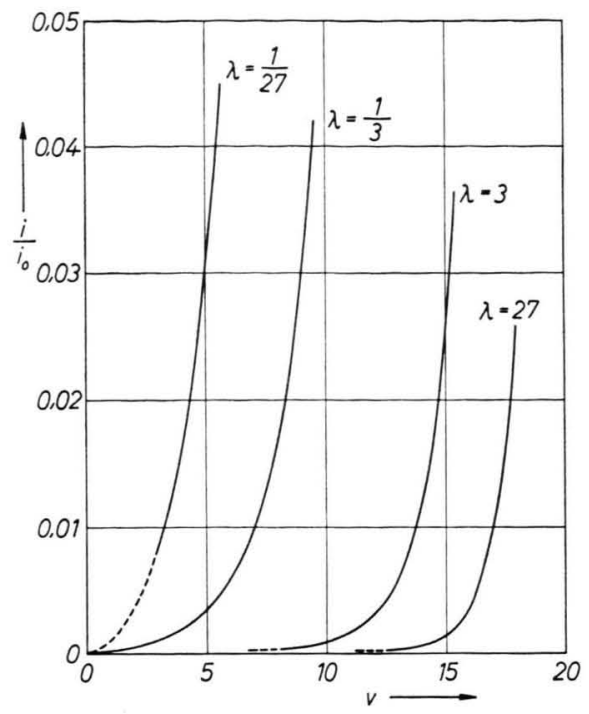

Abb. 6. Die Stromdichte $i$ als Funktion der reduzierten Spannung $v$ (Parameter $\left.\lambda=L / w_{0}\right)$.

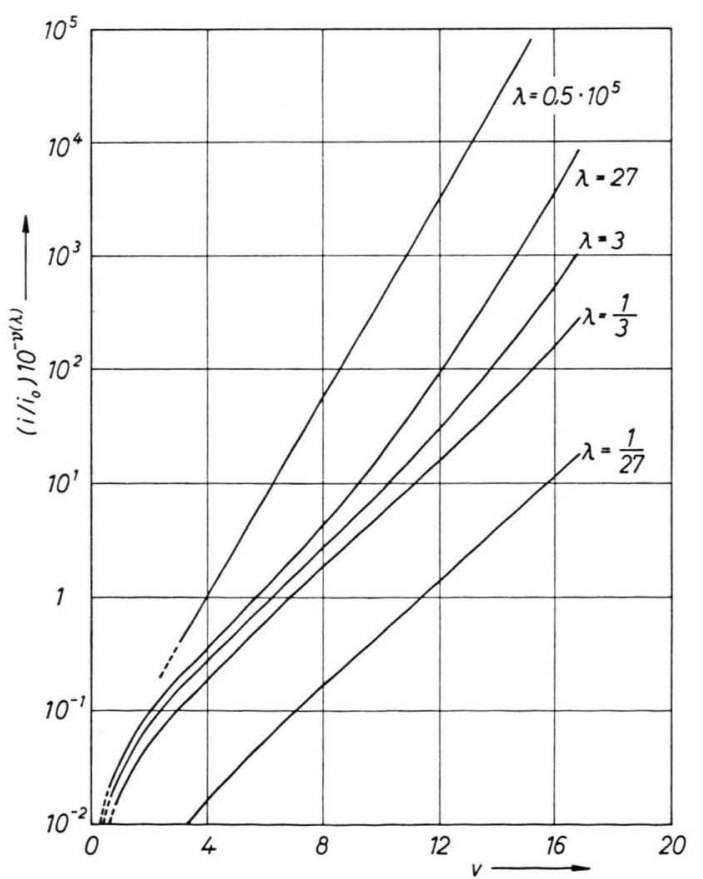

Abb. 7. Die Stromdichte $i$ als Funktion der reduzierten Spannung $v$ in logarithmischer Darstellung (Parameter $\lambda=L / w_{0}$ ).

\section{c) Diskussion der Ergebnisse}

Die bisher existierenden Arbeiten ${ }^{1-5}$ zur Theorie des $\mathrm{p}-\mathrm{n}$-Übergangs haben einen begrenzten Gültig- 
keitsbereich. Insbesondere kann die Annahme konstanter elektrochemischer Potentiale ${ }^{2,3}$ im Übergangsgebiet nur für hinreichend große Diffusionslängen $\lambda$ erfüllt sein. In der vorliegenden Arbeit

\begin{tabular}{|c|c|l|c|}
\hline$v$ & $\begin{array}{c}d \\
{\left[10^{-11}\right]}\end{array}$ & $\begin{array}{c}r \\
{\left[10^{-11}\right]}\end{array}$ & $\begin{array}{c}d+r \\
{\left[10^{-11}\right]}\end{array}$ \\
\hline 0,60 & 0,0164 & 0,0006 & 0,0170 \\
1,20 & 0,0464 & 0,0014 & 0,0478 \\
2,10 & 0,143 & 0,003 & 0,146 \\
3,00 & 0,382 & 0,006 & 0,388 \\
4,20 & 1,31 & 0,01 & 1,32 \\
6,30 & 10,9 & $0,0(2)$ & 10,9 \\
8,10 & 65,9 & & 65,9 \\
10,80 & 980 & & 980 \\
12,60 & 5930 & & 5930 \\
14,40 & 35900 & & 35900 \\
16,20 & 217000 & & 217000 \\
\hline
\end{tabular}

Tab. 4. $d, r, d+r$ als Funktion von $v ; \lambda=0,5 \cdot 10^{5}$.

\begin{tabular}{|c|c|c|c|}
\hline$v$ & $\begin{array}{c}d \\
{\left[10^{-6}\right]}\end{array}$ & $\begin{array}{c}r \\
{\left[10^{-6}\right]}\end{array}$ & $\begin{array}{c}d+r \\
{\left[10^{-6}\right]}\end{array}$ \\
\hline 0,60 & 0,0003 & 0,0202 & 0,0205 \\
1,20 & 0,0009 & 0,0465 & 0,0474 \\
2,10 & 0,003 & 0,102 & 0,105 \\
3,00 & 0,007 & 0,190 & 0,197 \\
4,20 & 0,024 & 0,396 & 0,420 \\
6,30 & 0,20 & 1,28 & 1,48 \\
8,10 & 1,22 & 3,44 & 4,66 \\
10,80 & 18,2 & 15,3 & 33,5 \\
12,60 & 110 & 41 & 151 \\
14,40 & 664 & 115 & 779 \\
16,20 & 4020 & 330 & 4350 \\
\hline
\end{tabular}

Tab. 5. $d, r, d+r$ als Funktion von $v ; \lambda=27$.

\begin{tabular}{|c|c|c|c|}
\hline$v$ & $d$ & $r$ & $d+r$ \\
\hline 3,25 & & 0,0101 & 0,0101 \\
4,50 & & 0,0227 & 0,0227 \\
5,80 & & 0,0492 & 0,0492 \\
6,89 & & 0,0907 & 0,0907 \\
8,16 & & 0,186 & 0,186 \\
10,34 & & 0,593 & 0,593 \\
12,20 & 0,00 & 1,56 & 1,56 \\
15,00 & 0,02 & 6,57 & 6,59 \\
16,88 & 0,1 & 16,9 & 17,0 \\
\hline
\end{tabular}

Tab. 6. $d, r, d+r$ als Funktion von $v ; \lambda=1 / 27$.

stellten wir uns die Frage, wo die Grenze für die Anwendbarkeit der Überlegungen von BERNARD ${ }^{2}$ und $\mathrm{S}_{\mathrm{AH}}$ u. a. ${ }^{3}$ zu ziehen sei und machten uns zur Aufgabe, das Aussehen der Strom - Spannungs-Charakteristiken über diese Grenze hinaus zu untersuchen. Wir verfolgten dieses Ziel, indem wir zunächst einen allgemeinen Formalismus zur Theorie des symmetrischen $\mathrm{p}-\mathrm{n}$-Übergangs entwickelten. Um zu quantitativen Aussagen zu kommen, schlossen wir die numerische Behandlung eines speziellen Beispiels an. Die gewonnenen Ergebnisse wollen wir nun im Hinblick auf die unserer Arbeit zugrunde liegende Fragestellung diskutieren.

Gehen wir von den Abbildungen 3 und 4 aus, so finden wir eine Antwort auf die Frage nach dem Gültigkeitsbereich der obengenannten Theorien ${ }^{2,3}$. Wie wir früher gezeigt haben, ist Voraussetzung für ihre Anwendbarkeit die Beziehung $\alpha=1$. Aus dem Verlauf der berechneten Kurven können wir entnehmen, daß diese Beziehung für $\lambda \geqq 1$ in guter Näherung erfüllt ist. Wir können in solchen Fällen $\alpha v=v$ setzen. Der auftretende Fehler wird für $\lambda=1$ am größten; es gilt $v-\alpha v<0,2$, so daß die $\mathrm{Ab}$ weichung des durch $e^{\alpha v}$ bestimmten Stromes vom exakten Wert $20 \%$ betragen kann. Wir kommen also für unser spezielles Beispiel zu dem Schluß, daß die Arbeiten von Bernard ${ }^{2}$ und $\mathrm{S}_{\mathrm{AH}}$ u. a. ${ }^{3}$ gelten, solange die Diffusionslänge größer ist als die Breite des Übergangsgebietes im Gleichgewicht.

In Abb. 5 haben wir $\alpha v$ als Funktion von $v$ dargestellt. Für $\lambda \geqq 1$ gilt nach den vorangegangenen Überlegungen $\alpha v \simeq v$, wir erhalten eine Gerade durch den Nullpunkt. Für Diffusionslängen $\lambda<1$ ergeben sich Kurven, die bei hinreichend großen Spannungen in guter Näherung durch

$$
\alpha v=v-v_{0}, \quad v_{0}>0
$$

beschrieben werden können, also auch linear sind. Ihre Steigung hat bis auf Abweichungen von wenigen Prozent den Wert 1 in Übereinstimmung mit der Geraden $\alpha v=v$. Es ist bemerkenswert, da $\beta$ alle dargestellten Kurven mit wachsender Spannung in Parallelen übergehen.

Die ermittelten Strom - Spannungs-Charakteristiken sind in den Abb. 6 und 7 dargestellt. Es sei nochmals darauf hingewiesen, daß die Stromdichteneinheit für jede der Kurven in Abb. 7 eine andere ist, da der Exponent $v$ von der Diffusionslänge $\lambda$ abhängt, während wir in Abb. 6 übereinstimmend die Einheit $i_{0}$ gewählt haben. Aus den Tabellen 4, 5 und 6 ist ersichtlich, daß bei großen Diffusionslängen der Diffusionsanteil $d$ den Rekombinationsanteil $r$ des Stromes überwiegt und daß mit abnehmender Diffusionslänge der Rekombinationsstrom zunehmend an Bedeutung gewinnt. Insbesondere gilt $i / i_{0} \simeq d$ für $\lambda=0,5 \cdot 10^{5}$, während für $\lambda=\frac{I}{27}$ $i / i_{0} \simeq r$ wird. Diese Erscheinung wird auch in Abb. 7 deutlich. Der Diffusionsanteil wird durch den Faktor $e^{\alpha v}$, der Rekombinationsanteil durch $e^{\alpha v / 2}$ in sei- 
ner Spannungsabhängigkeit bestimmt. Nach (65) folgt $e^{x v} \sim e^{v}$ und $e^{x v / 2} \sim e^{v / 2}$ für hinreichend große $v$, so daß die zu $\lambda=0,5 \cdot 10^{5}$ und $\lambda=1 / 27$ gehörigen Charakteristiken in logarithmischer Darstellung als Geraden mit um einen Faktor 2 verschiedenen Steigungen erscheinen. Betrachten wir eine Kurve, deren zugeordnete Diffusionslänge zwischen den eben diskutierten Grenzfällen liegt, so sehen wir, daß die Bedeutung des Diffusionsstromes mit zunehmender Spannung zu-, die des Rekombinationsstromes dagegen abnimmt, was ebenfalls in der sich ändernden Steigung zum Ausdruck kommt. Das Ergebnis, daß für kleine Diffusionslängen und genügend große Spannungen $i \sim e^{v / 2}$ wird, steht in Übereinstimmung mit den Überlegungen von $\mathrm{MA}_{\mathrm{A}}$ DELUNG $^{5}$ für den Grenzfall $\lambda=0$ und zeigt, daß diese Beziehung sich nicht nur bei Voraussetzung konstanter elektrochemischer Potentiale im Übergangsgebiet $^{2-4}$ ergibt; das ist eine Folge von Gl. (65).

Herrn Prof. Dr. O. Madelung danke ich für sein förderndes Interesse an der Arbeit, wertvolle Ratschläge und Diskussionen sowie Herrn Obering. H. Hoffmans für sein freundliches Entgegenkommen bei der Berechnung von Integralen auf einer Rechenanlage der Siemens-Schuckertwerke in Erlangen. Mein Dank gilt auch der Deutschen Forschungsgemeinschaft, die den Start der Arbeit durch eine Sachbeihilfe unterstützt hat.

\title{
Aktivierungsenergie und Aktivierungsvolumen bei kleinen Spannungen und Verformungsgeschwindigkeiten
}

\author{
Von Georg Alefeld \\ Aus dem Laboratorium für Technische Physik der Technischen Hochschule München \\ (Z. Naturforschg. 17 a, 899-905 [1962]; eingegangen am 14. Juli 1962)
}

\begin{abstract}
Bei kleinen Spannungen und Verformungsgeschwindigkeiten oder höheren Temperaturen müssen die Formeln, durch deren Anwendung man aus sprungartigen Änderungen der Versuchsbedingungen bei der Verformung von Metallen Aktivierungsenergie und Aktivierungsvolumen bestimmt, modifiziert werden. Mit Hilfe der korrigierten Formeln finden einige neuere experimentelle Ergebnisse eine zwanglose Deutung.
\end{abstract}

Die experimentellen und theoretischen Arbeiten von Basinski ${ }^{1}$, Conrad und Wiedersich ${ }^{2}$, Sherby, Lytton und Dorn ${ }^{3}$, Conrad ${ }^{4}$, Conrad, Hays, Schoeck und Wiedersich ${ }^{5}$, Conrad und Robertson ${ }^{6,7}$, Feltham und Meakin $^{8}$ usw. zeigen, wie man bei plastischer Verformung von Festkörpern durch sprungartige Änderung von Temperatur, Spannung oder Verformungsgeschwindigkeit Aussagen über die den Verformungsmechanismus kennzeichnenden Größen Aktivierungsenergie und Aktivierungsvolumen erhält.

Die Auswertung der Messungen beruht auf folgendem Ansatz für die Verformungsgeschwindigkeit $\dot{\varepsilon}$ :

$$
\text { mit } \quad v=\varepsilon_{0} v_{0} N e^{S / k} \text {. }
$$

$v$ ist das Produkt aus dem Frequenzfaktor $v_{0}$, der

1 Z. S. Basinski, Acta Met. 5, 684 [1957].

2 H. Conrad u. H. Wiedersich, Acta Met. 8, 128 [1960].

3 O. D. Sherby, J. L. Lytton u. J. E. Dorn, Acta Met. 5, 219 [1957].

4 H. Conrad, Acta Met. 6, 339 [1958].

5 H. Conrad, L. Hays, G. Schoeck u. H. Wiedersich, Acta Met. 9, 367 [1961]. makroskopischen Verformung $\varepsilon_{0}$, die sich bei der Überwindung eines Hindernisses durch ein Versetzungselement ergibt, der Zahl der aktivierbaren Elemente $N$ und einem Faktor $e^{S / k}$, in $\operatorname{dem} S$ die Aktivierungsentropie darstellt. $H$ ist die Aktivierungsenthalpie, die meist als Aktivierungsenergie bezeichnet wird. $\sigma$ ist die effektive Spannung, also die Differenz aus äußerer und innerer Spannung, d. h.

$$
\sigma=\sigma_{\mathrm{a}}-\sigma_{\mathrm{i}}
$$

$v \sigma$ stellt die Arbeit dar, die die Spannung $\sigma$ bei der Überwindung der Hindernisse durch die Versetzung leistet. $v$ hat die Dimension eines Volumens und wird als Aktivierungsvolumen bezeichnet ${ }^{9}$. Es ist ebenfalls der Ausdruck Spannungskonzentrationsfaktor gebräuchlich ${ }^{8}, 10$.

${ }^{6}$ H. Conrad u. W. D. Robertson, Trans. Amer. Inst. Min. Metall Engrs. 209, 503 [1957].

7 H. Conrad u. W. D. Robertson, Trans. Amer. Inst. Min. Metall Engrs. 212, 536 [1958].

8 P. Feltham u. J. D. Meakin, Acta Met. 7.614 [1959].

9 A. Seeger, Handbuch der Physik, Bd. VII/2, Verlag Springer, Berlin 1958.

10 P. Feltham, Phil. Mag. 2, 584 [1957]. 